\title{
Measurement and Statistical Analysis of Distinguishable Multipaths in Underground Tunnels
}

\author{
Junchang Sun $\mathbb{D}^{1},{ }^{1}$ Shuai Ma $\mathbb{D}^{1,2,3}$ Hui Zhou ${ }^{1}{ }^{1},{ }^{1}$ Chun Du $\mathbb{D}^{1},{ }^{1}$ and Shiyin Li $\mathbb{D}^{1}$ \\ ${ }^{1}$ School of Information and Control Engineering, China University of Mining and Technology, Xuzhou 221116, China \\ ${ }^{2}$ National Mobile Communications Research Laboratory, Southeast University, Nanjing 211189, China \\ ${ }^{3}$ Shaanxi Key Laboratory of Information Communication Network Security, Xi'an University of Posts \& Telecommunications, \\ Xi'an 710121, China \\ Correspondence should be addressed to Shiyin Li; lishiyin@cumt.edu.cn
}

Received 27 July 2020; Revised 29 October 2020; Accepted 21 November 2020; Published 7 December 2020

Academic Editor: Lorenzo Luini

Copyright (c) 2020 Junchang Sun et al. This is an open access article distributed under the Creative Commons Attribution License, which permits unrestricted use, distribution, and reproduction in any medium, provided the original work is properly cited.

\begin{abstract}
Compared with the line-of-sight (LOS) condition, the multipath effect is more serious in the non-line-of-sight (NLOS) condition. Therefore, the LOS and NLOS identification is necessary for the multipath analysis of signal propagation. The commonly used method is the support vector machine (SVM) method with high computational complexity. To tackle this problem, this paper adopts the SVM classifier based on fewer selected features of the normalized power delay profile (PDP). Therein, the PDP can be obtained using the sliding correlation method. The results show that the SVM-based classifier can achieve high accuracy on LOS and NLOS identification. We then analyze the impact of the signal-to-noise ratio (SNR) and transmitting-receiving (Tx-to-Rx) distance on distinguishable multipaths under LOS and NLOS conditions. According to statistical measurement results, a function of distinguishable multipath numbers is established. Finally, we investigate the multipath power and delay parameters of average delay spread and root mean square (RMS) delay spread based on multipath results. The outcomes of this paper provide a useful support for analyzing signal propagation characteristics.
\end{abstract}

\section{Introduction}

Communication systems can be developed in various environments such as tunnels instead of being limited to free space $[1,2]$. Therefore, it is necessary to explore signal propagation characteristics in narrow spaces $[3,4]$.

Due to the confined space of a tunnel environment, internal wireless signals are susceptible to be interfered caused by non-line-of-sight (NLOS) effects, resulting in amplitude attenuation and phase shift of signal propagation $[5,6]$. Therefore, it is significant to identify line-of-sight (LOS) and NLOS conditions. The ranging-based estimation methods are investigated in [7-9], and the power-based methods are adopted in $[10,11]$. Additionally, deep learning methods are used for identification. For example, an overview of opportunities and challenges of the deep learning-based classification method is presented in [12]. The multimodal deep learning method is proposed to design the mobile encrypted traffic classifier based on automatically extracted features [13].

Moreover, the LOS and NLOS conditions are identified in several systems. For example, for the ultrawideband (UWB) system, the support vector machines (SVMs) are developed in [14-16], with the results showing that the NLOS condition can be effectively identified based on UWB signal features. In [17], the multipath condition is added to be considered, i.e., the machine learning techniques are compared to identify LOS, NLOS, and multipath conditions, with the results showing the effectiveness of the machine learning method in the field of the UWB indoor localization. For the vehicle-to-vehicle (V2V) system, in [18], the SVMbased method is used via combining the angular information and conventional channel features to identify LOS and NLOS conditions. In [19], the machine learning-based LOS identification methods are compared based on angular properties of the dynamic channel impulse response (CIR), 
which effectively improves the identification accuracy. Inspired by these, we aim to investigate the LOS and NLOS identification method for the tunnel environment.

In addition to LOS and NLOS identification, a considerable amount of research has been conducted on the characteristics of wireless channels in tunnels. For example, the $2.4 \mathrm{GHz}$ band signal is used for a subway in Madrid, and the arrival time distribution of multipath delay clusters is analyzed under LOS, NLOS, and far-line-of-sight (FLOS) conditions [20]. The measurement results of [21] provide insights into the large-scale fading characteristics in actual curved subway tunnels with distances of $300 \mathrm{~m}$ and $500 \mathrm{~m}$ at $920 \mathrm{MHz}, 2.4 \mathrm{GHz}$, and $5.7 \mathrm{GHz}$. Zhou et al. [22] investigated measurement data on radio frequency (RF) propagation in tunnel and mine environments for vertically, horizontally, and cross-polarized signals; their results showed the importance of tunnel size, frequency, polarization, and electrical characteristics of the tunnel wall on signal propagation characteristics. Radio channel characteristics such as path loss, root mean square (RMS) delay spread, channel stationarity, Doppler shift, and channel capacity are investigated in a subway tunnel [23].

In this paper, we adopt the SVM-based method to identify LOS and NLOS conditions for the tunnel environment. Compared with the used SVM in $[14,16]$, we only select variance, mean excess delay, root mean square (RMS) delay spread, and kurtosis of the normalized power delay profile (PDP) as identification features. The main contributions of our works are outlined as follows:

(i) We only select four features to identify LOS and NLOS conditions with the SVM method for the tunnel environment, which significantly reduces the computational complexity.

(ii) We analyze the change in distinguishable multipaths by comparing the normalized PDP of measurement data under LOS and NLOS conditions. Moreover, a function of distinguishable multipath numbers is established according to the statistical results. Additionally, the multipath power and delay parameters are analyzed under the LOS and NLOS conditions.

(iii) All measurement data are available to be downloaded at https://github.com/sunjc-cumt/measurementdata. To the best of our knowledge, this is valuable to provide downloadable actual measurement data for the tunnel environment.

The rest of this paper is organized as follows. In Section 2, the system model is presented. In Section 3, the SVM classifier and identification method are presented. In Section 4 , the design of the measurement system and the measurement scenarios are described. In Section 5, the measurement results are analyzed. Finally, conclusions are presented in Section 6.

Notations: the notations $a$, a, and A denote the scalar, vector, and matrix, respectively. The operator $*$ represents the convolution. $\mathbf{A}^{T}$ denotes the transpose of matrix $\mathbf{A} .|\cdot|$ and $\|\cdot\|_{2}$ denote the absolute value and $\ell_{2}$-norm, respectively. $\lceil x\rceil$ denotes the smallest integer greater than $x$. $\mathbb{E}(\cdot)$ denotes the expectation operator, and $\operatorname{sign}(\cdot)$ denotes the sign function. $\mathscr{N} \triangleq\{1,2, \ldots, N\}$.

\section{System Model}

Due to the confined space of a tunnel environment, obvious refraction and reflection phenomena occur during signal propagation [24], as shown in Figure 1. The transmitted signal travels multiple paths to the receiver. The general formulation of a CIR in a tunnel environment [25] can be given by

$$
h(t)=\sum_{l=0}^{L-1} \alpha_{l} \delta\left(t-\tau_{l}\right) e^{j \theta_{l}}
$$

where $L$ represents the total distinguishable multipath numbers and $\alpha_{l}, \tau_{l}$, and $\theta_{l}$ represent the amplitude, delay, and phase of the $l$-th path, respectively. The phase $\theta_{l}$ is assumed to be a priori, statistically independent uniform random variable distributed over $[0,2 \pi)$ [26].

PDP is important in the analysis of channel characteristics, which can be obtained by the sliding correlation method. The transmitted signal $x(t)$ is assumed to be an $m$ sequence modulated by QPSK. By not considering the phase factor in (1), the received signal can be given as

$$
y(t)=h(t) * x(t)+n(t)=\sum_{l=0}^{L-1} \alpha_{l} x\left(t-\tau_{l}\right)+n(t),
$$

where $n(t)$ denotes the additive white Gaussian noise (AWGN). The received signal is correlated with the known transmitted signal by using the sliding correlation method. Based on the stable autocorrelation of an $m$-sequence, we can obtain

$$
h(t)=R_{x y}(t)=\sum_{l=0}^{L-1} \alpha_{l} R_{x x}\left(t-\tau_{l}\right),
$$

where $R_{x y}(t)$ represents a cross-correlation function between the received and transmitted signals and $R_{x x}(t)$ represents an autocorrelation function of $x(t)$. A correlation function profile consisting of multiple peaks is obtained, which is known as the PDP. Subsequently, the channel characteristics can be represented by PDPs.

\section{LOS and NLOS Identification}

The SVM classifier is adopted to identify LOS and NLOS conditions based on selected features. For reducing computational complexity, we only select variance, mean excess delay, RMS delay spread, and kurtosis of the normalized PDP as identification features. The form of the SVM classifier is given by

$$
z(\boldsymbol{v})=\operatorname{sign}(g(\boldsymbol{v}))
$$

where

$$
g(v)=\mathbf{w}^{T} \phi(v)+b .
$$




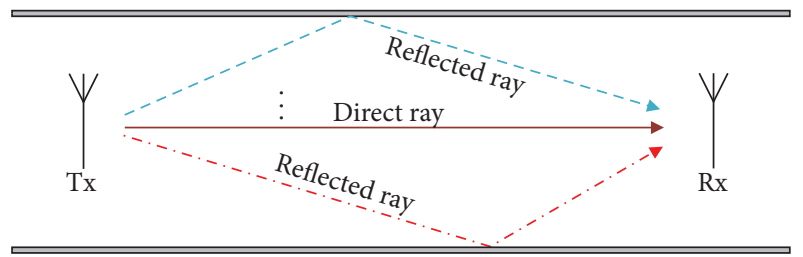

FIGURE 1: Signal propagation in a tunnel environment.

$\mathbf{w}$ and $b$ are unknown parameters which are determined based on $\left\{v_{i}, z_{i}\right\}, i \in \mathcal{N}, N$ is the number of reference points, and $v_{i} \in \mathbb{R}^{4}$ denotes the selected features, i.e., variance, mean excess delay, RMS delay spread, and kurtosis, and $\phi(\cdot)$ is a predetermined function, and $z_{i} \in\{-1,1\}$ with

$$
z_{i}= \begin{cases}1, & \text { LOS, } \\ -1, & \text { NLOS. }\end{cases}
$$

The SVM classifier aims to maximize the margin $\left(2 /\|\mathbf{w}\|_{2}\right)$ with slack between two classes [27], which is presented as

$$
\begin{array}{ll}
\min _{\mathbf{w}, b, \xi} & \frac{1}{2}\|\mathbf{w}\|_{2}^{2}+\zeta \sum_{i \in \mathcal{N}} \xi_{i} \\
\text { s.t. } & z_{i}\left(\mathbf{w}^{T} \phi\left(\boldsymbol{v}_{i}\right)+b\right) \geq 1-\xi_{i}, \quad i \in \mathcal{N}, \\
& \xi_{i} \geq 0, \quad i \in \mathcal{N},
\end{array}
$$

where $\xi_{i}$ are slack variables and $\zeta>0$ is the penalty term.

Generally, the Lagrangian dual of the optimization (7) is a quadratic program (QP), which can be solved with the sequential minimal optimization (SMO) method [28, 29]. However, for complexity consideration, this method is usually replaced by the least squares (LS) method [30]. The LS-SVM is used to turn the inequality constraint into an equality constraint, which facilitates the solution of the Lagrange multiplier. Consequently, the problem is a linear equation in the LS-SVM:

$$
\begin{array}{ll}
\min _{\mathbf{w}, b, \xi} & \frac{1}{2}\|\mathbf{w}\|_{2}^{2}+\frac{1}{2} \zeta \sum_{i \in N} \Delta_{i}^{2} \\
\text { s.t. } & z_{i}\left(\mathbf{w}^{T} \phi\left(\boldsymbol{v}_{i}\right)+b\right)=1-\Delta_{i}, \quad i \in \mathcal{N},
\end{array}
$$

where $\Delta_{i}$ denotes the error.

Based on the principle of the LS-SVM, the method of the identification of LOS and NLOS conditions is given in Algorithm 1. In the training phase, the Lagrangian dual of optimization problem (8) is given by

$$
\begin{aligned}
\mathscr{L}(\mathbf{w}, b, \Delta, \mu)= & \frac{1}{2}\|\mathbf{w}\|_{2}^{2}+\frac{1}{2} \zeta \sum_{i \in \mathcal{N}} \Delta_{i}^{2} \\
& -\sum_{i \in \mathcal{N}} \mu_{i}\left(z_{i}\left(\mathbf{w}^{T} \phi\left(\mathbf{v}_{i}\right)+b\right)-1+\Delta_{i}\right),
\end{aligned}
$$

where $\mu_{i}$. denotes Lagrange multiplier. Then, by setting partial derivatives of the Lagrangian dual $\mathscr{L}$ to parameters $\mathbf{w}$, $b, \Delta$, and $\mu$ to zero, we can obtain values of $\mu_{i}, b$, and

$$
\mathbf{w}=\sum_{i \in \mathcal{N}} \mu_{i} z_{i} \phi\left(\boldsymbol{v}_{i}\right)
$$

In the testing phase, based on trained parameters, we can obtain the identification result for the given testing data $\widetilde{v}$, which is given by

$$
z(\widetilde{v})=\operatorname{sign}\left(\sum_{i \in \mathcal{N}} \mu_{i} z_{i} \kappa\left(\widetilde{v}, v_{i}\right)+b\right),
$$

where $\kappa\left(\widetilde{\nu}, v_{i}\right)=\phi(\widetilde{v})^{T} \phi\left(v_{i}\right)$ denotes the kernel function. Typically, the linear, poly, Gaussian, and radial basis function (RBF) kernel functions are adopted to solve (11).

\section{Measurement System and Environment}

4.1. Measurement System. In this section, we describe the measurement system and environment. The designed measurement system is shown in Figure 2.

In this study, we adopt a RF vector signal generator (N5172B), which can generate $2.4 \mathrm{GHz}$ Wi-Fi to transmit signals and adopt a vector signal analyzer (N9020B) to receive signals. Additionally, we use a BNC line as trigger to connect the transmitting and receiving instruments for time synchronization.

The transmitted signal ( $m$-sequence) and trigger signal are imported into N5172B through processor-side Toolkit software and sent through the transmitting antenna after setting transmission power and sample rate. Then, N9020B receives and stores signals through the receiving antenna after time synchronization. It is worth pointing out that the directions of the receiving antenna and the transmitting antenna are always aligned throughout the experiment. Finally, we use the sliding correlation method to obtain PDPs at the processor side. The parameters of the transmitting and receiving antennas are summarized in Table 1.

4.2. Measurement Environment. A typical underground tunnel environment is applied as the measurement environment. The tunnel is arched with closed glass doors at both ends and two ramps of the same size in the middle section.

Figure 3(a) shows a long straight tunnel, wherein the width of both ramps is $5.8 \mathrm{~m}$. The other side of the ramp is an auxiliary road with a width of $1 \mathrm{~m}$; it runs parallel to the tunnel. Figure 3(b) shows an arched cross section with a side height of $2.3 \mathrm{~m}$, a vault height of $3.6 \mathrm{~m}$, and a width of $3 \mathrm{~m}$. The measurement setup is shown in Figure 3(c).

For convenience of position description, each test point in Figure 3(a) is given in the form of two-dimensional coordinates. Tx1 is taken as the reference point, and the coordinate is set to $(0,0)$ in meters. The coordinates of reference points are listed in Table 2.

\section{Analysis of Measurement Results}

5.1. LOS and NLOS Identification. We measure received signals at the given Rx points under LOS and NLOS conditions. In order to ensure the accuracy of the analysis, we 
Input: training set $\left\{v_{i}, z_{i}\right\}, i \in \mathcal{N}$,

Testing data $\widetilde{v}$.

Output: identification results.

IITraining phase:

(1) Obtain the Lagrangian dual $\mathscr{L}(\mathbf{w}, b, \Delta, \mu)$ based on equation (9);

(2) Set partial derivatives of the Lagrangian dual $\mathscr{L}$ to parameters $\mathbf{w}, b, \Delta$, and $\mu$ to zero;

(3) We can obtain

$$
\left\{\begin{array}{l}
(\partial \mathscr{L} / \partial \mathbf{w})=0 \Longrightarrow \mathbf{w}=\sum_{i \in \mathcal{N}} \mu_{i} z_{i} \phi\left(v_{i}\right) \\
(\partial \mathscr{L} / \partial b)=0 \Longrightarrow \sum_{i \in \mathcal{N}} \mu_{i} z_{i}=0 \\
\left(\partial \mathscr{L} / \partial \Delta_{i}\right)=0 \Longrightarrow \mu_{i}=\zeta \Delta_{i}, \quad i \in \mathscr{N} \\
\left(\partial \mathscr{L} / \partial \mu_{i}\right)=0 \Longrightarrow z_{i}\left(\mathbf{w}^{T} \phi\left(\nu_{i}\right)+b\right)+\Delta_{i}-1=0, \quad i \in \mathscr{N}
\end{array}\right.
$$

IITesting phase:

(4) For the given testing data $\widetilde{\nu}$, we calculate the output $z(\widetilde{\nu})$ based on equation (11);

(5) Identify LOS and NLOS conditions based on equation (6).

Algorithm 1: LOS and NLOS identification with the LS-SVM.

\begin{tabular}{|c|c|c|}
\hline Parameters & Tx-antenna & Rx-antenna \\
\hline Frequency (GHz) & $2.4-2.5$ & $2.4-2.5$ \\
\hline Type of polarization & $\begin{array}{c}\text { Vertical } \\
\text { polarization }\end{array}$ & $\begin{array}{c}\text { Vertical } \\
\text { polarization }\end{array}$ \\
\hline Antenna gain $(\mathrm{dBi})$ & 12 & 6 \\
\hline Width of the lobe ${ }^{(\circ)}$ & Vertically: 14 & Vertically: 25 \\
\hline Standing wave ratio & $\leq 1.5$ & $\leq 1.5$ \\
\hline Impedance $(\Omega)$ & 50 & 50 \\
\hline Maximum power $(\mathrm{W})$ & 100 & 50 \\
\hline $\begin{array}{l}\text { Weight of the antenna } \\
(\mathrm{kg})\end{array}$ & 3 & 0.5 \\
\hline $\begin{array}{l}\text { Height of the antenna } \\
\text { (m) }\end{array}$ & 1.8 & 1.8 \\
\hline
\end{tabular}

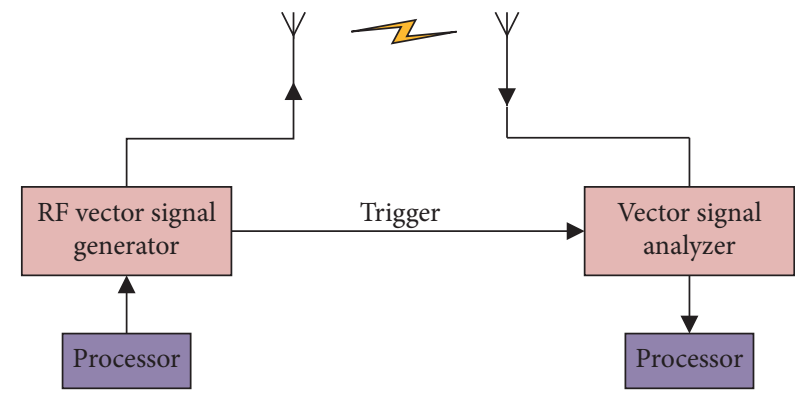

FIGURE 2: Transmitting and receiving system.

TABle 1: Antenna parameters.

collect $50 \%$ data under LOS conditions and 50\% data under NLOS conditions. Then, the SVM classifier is adopted to identify LOS and NLOS conditions. Figure 4 demonstrates the receiver operating characteristic (ROC) curve of four kernel functions on identification. The black dashed line is the identification ability baseline, i.e., there is no identification ability in this line. The result shows that the SVM classifier with four kernel functions has a high accuracy on identifying LOS and NLOS conditions.

Numerically, we present the area under the ROC curve (AUC) values as shown in Table 3. The results intuitively present that the AUC values can be up to 0.96 and 0.97 .
Therefore, each kernel function can be adopted to aid the SVM classifier for identification and has a high accuracy.

The computational complexity of the SVM classifier is divided into the training phase and testing phase. In the training phase, generally, we have $\left(N_{\text {sv }} / N\right) \ll 1$, and the computational complexity is $O\left(N_{\mathrm{sv}}^{2}+M N_{\mathrm{sv}} N\right)$, where $M$ is the dimension of the selected feature set, $N_{\mathrm{sv}}$ is the number of the support vector, and $N$ is the number of reference points. In the testing phase, the computational complexity is $O\left(V N_{\text {sv }}\right)$, where $V$ denotes the number of operations required to evaluate the kernel. Therefore, the total computational complexity is $O\left(N_{\mathrm{sv}}^{2}+M N_{\mathrm{sv}} N\right)+O\left(V N_{\mathrm{sv}}\right)$ [31]. In comparison to previous works $[14,16]$, we only select four features in our works, i.e., $M=4$, and the dimension of the feature set is lower. Therefore, the computational complexity is obviously reduced.

5.2. Analysis of Distinguishable Multipaths. In this section, the effects of various factors on the distinguishable multipaths are analyzed under LOS and NLOS conditions. It is worth pointing out that the secondary peak can be considered as a distinguishable multipath component when the peak value is within $30 \mathrm{~dB}$ (refer to recommendation ITU-R P.1407-4 [32]; the threshold used for the identification of the number of multipath components depends on the dynamic range of the measurement equipment; a typical value is $20 \mathrm{~dB}$ in the outdoor environment. According to our measurement environment, equipment, and data, the threshold is set to $30 \mathrm{~dB}$ in this paper) lower than that of the main peak in each PDP.

5.2.1. LOS Conditions. The receiver can receive direct signals from the transmitter under LOS conditions. Some examples are presented: (i) the transmitting and receiving positions are set at Tx1 and $\mathrm{Rx} 7$, respectively, with a distance of $30 \mathrm{~m}$, as shown in Figure 3(a). The transmission power is set to $-10 \mathrm{dBm}$ and $0 \mathrm{dBm}$ to obtain two PDPs. The PDPs can be compared as shown in Figure 5(a). (ii) The transmission 


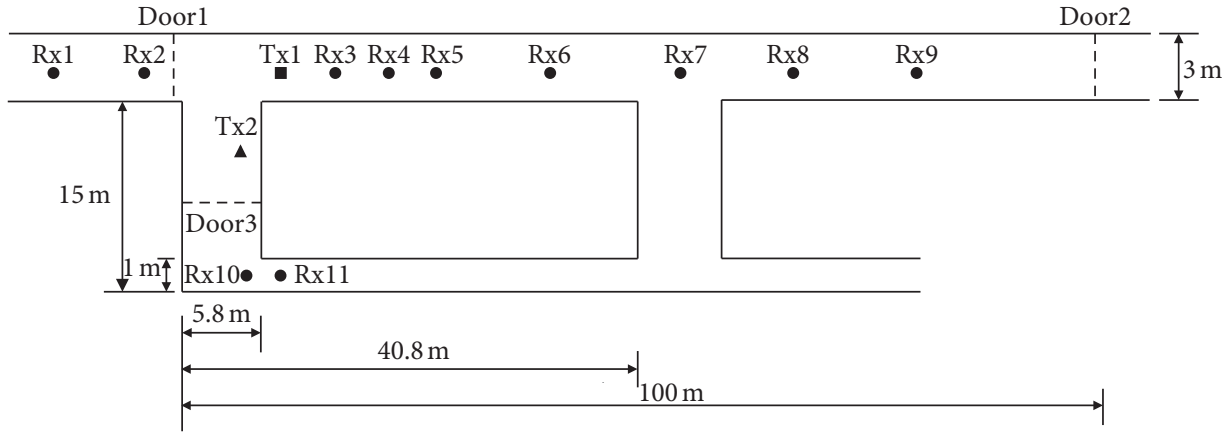

- Tx1 : Tx position in LOS

A Tx2 : Tx position in NLOS

- $\mathrm{Rx}$ : Rx positions

(a)

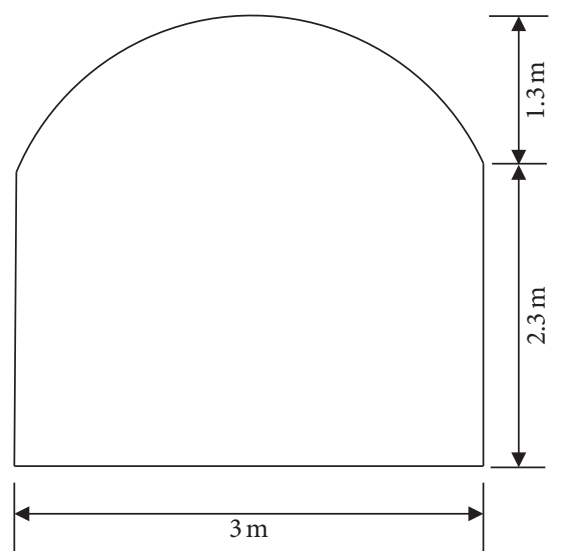

(b)

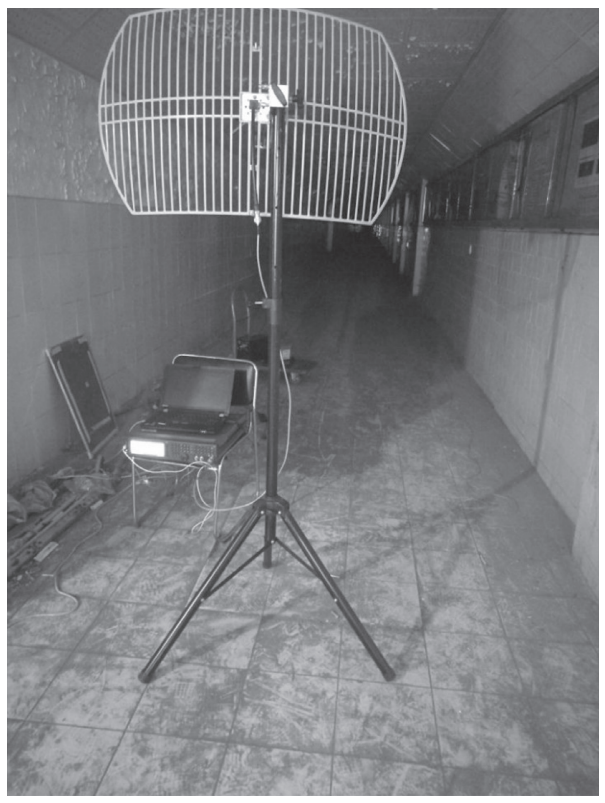

(c)

FIgURE 3: Measurement environment: (a) schematic; (b) arched cross section; (c) measurement setup. 
TABle 2: Coordinates of test points.

\begin{tabular}{lccc}
\hline Points & $(x, y)$ & Points & $(x, y)$ \\
\hline Tx1 & $(0,0)$ & $\mathrm{Rx} 6$ & $(20,0)$ \\
Tx2 & $(-2,5)$ & $\mathrm{R} 7$ & $(30,0)$ \\
$\mathrm{Rx} 1$ & $(-20,0)$ & $\mathrm{Rx} 8$ & $\mathrm{R} 9$ \\
$\mathrm{Rx} 2$ & $(-10,0)$ & $\mathrm{Rx} 10$ & $(50,0)$ \\
$\mathrm{Rx} 3$ & $(3,0)$ & $\mathrm{Rx} 11$ & $(-2,15)$ \\
$\mathrm{R} 4$ & $(8,0)$ & - & $(0,15)$ \\
$\mathrm{Rx} 5$ & $(10,0)$ & - \\
\hline
\end{tabular}

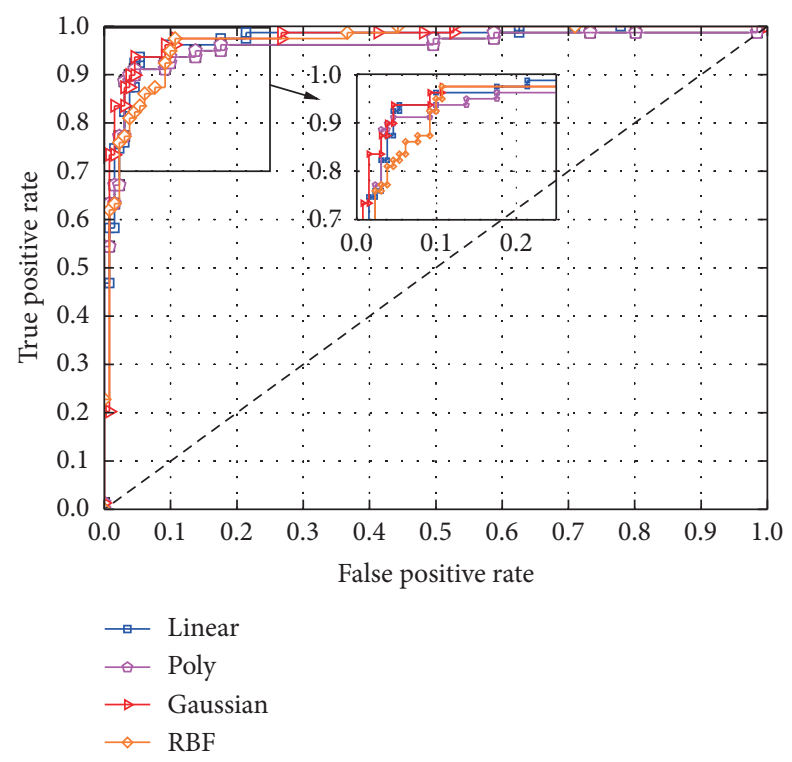

FIGURE 4: ROC of four kernel functions on identification.

TABLE 3: AUC values of four kernel functions.

\begin{tabular}{lcccc}
\hline Kernel function & Linear & Poly & Gaussian & RBF \\
\hline AUC & 0.97 & 0.96 & 0.97 & 0.96 \\
\hline
\end{tabular}

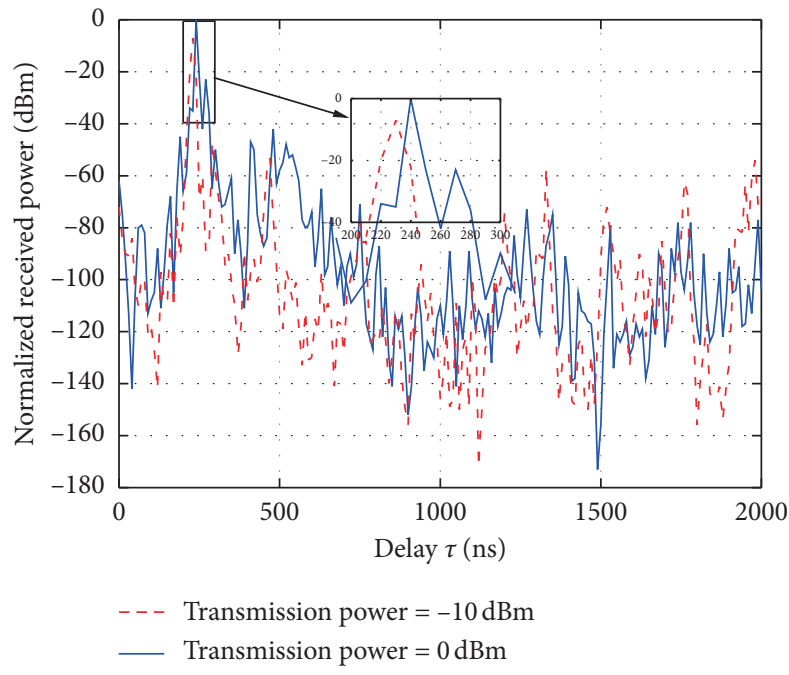

(a)

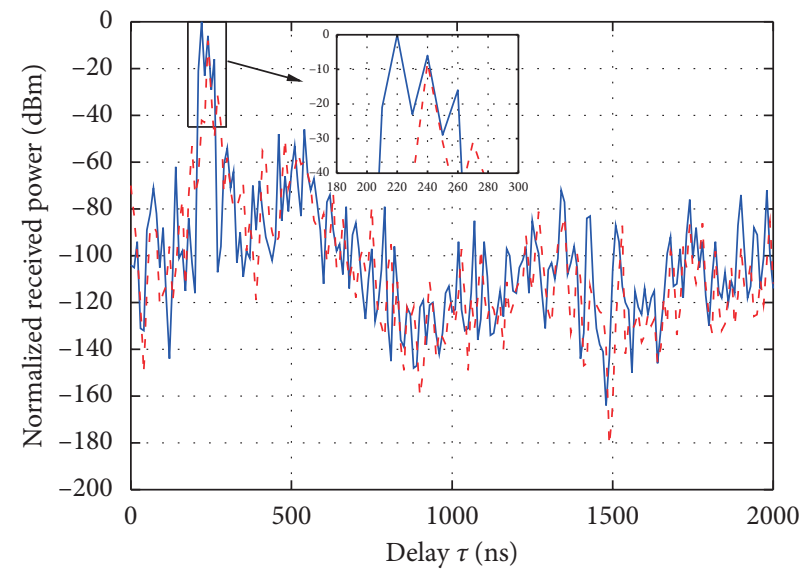

- - Distance $=30 \mathrm{~m}$

Distance $=40 \mathrm{~m}$

(b)

FIGURE 5: Comparison of PDPs under LOS conditions. (a) Different transmission powers. (b) Different distances. 
power is fixed to $0 \mathrm{dBm}$, and the receiver is placed at $\mathrm{Rx} 7$ and $\mathrm{Rx} 8$, while the transmitter is placed at Tx1. The change in the distinguishable multipath numbers is analyzed with respect to Tx-to-Rx distances, as shown in Figure 5(b).

As illustrated in Figure 5(a), only the direct signal can be distinguished, and the nondirect component cannot be distinguished when the transmission power is set to $-10 \mathrm{dBm}$ with receiving signal-to-noise ratio (SNR) being $8 \mathrm{~dB}$. However, the two components can be distinguished with a delay of $30 \mathrm{~ns}$ when the transmission power increases to $0 \mathrm{dBm}$ with receiving $\mathrm{SNR}$ being $17 \mathrm{~dB}$. It can be concluded that the nondirect components can be easily distinguished at the receiver with the SNR increasing.

Figure 5(b) shows that the two paths can be distinguished with a delay of $30 \mathrm{~ns}$ when the distance is $30 \mathrm{~m}$. The three paths can be distinguished with a delay of $20 \mathrm{~ns}$ and $40 \mathrm{~ns}$ when the distances increase to $40 \mathrm{~m}$. It can be concluded that when the transmission power is sufficiently high, the distinguishable multipath numbers increase with distances increasing within a certain range.

5.2.2. NLOS Conditions. The receiver can only receive refracted and reflected signals from the transmitter under NLOS conditions. Similarly, several examples are conducted under NLOS conditions: (i) the transmitter and receiver are placed at Tx2 and Rx3, respectively. The PDPs in the LOS and NLOS scenarios are compared the same distances, as shown in Figure 6(a). (ii) The transmitter is placed at $\mathrm{Tx} 2$, and the receiver is placed at $\mathrm{Rx} 5$ and $\mathrm{Rx} 6$, respectively. A comparison of the PDPs is shown in Figure 6(b) (for convenience, we mark the distance of the $x$-axis of Tx-to- $\mathrm{Rx}$ in Figure 6(b), and the real Tx-to-Rx distance can be calculated by coordinates in Table 2). (iii) The transmitter is placed at Tx1, while the receiver is placed at $\mathrm{Rx} 10$ and $\mathrm{Rx} 11$, respectively. The measurement paths look like " $L$ " and " $U$ " types, respectively, as shown in Figure 3(a). The multipath effects of two types of measurement conditions are compared and analyzed, as shown in Figure 6(c).

Figure 6 shows that, in the NLOS scenario, the distinguishable multipath numbers reduced compared with the LOS scenario with the same transmission power and distances. Furthermore, the effects of NLOS cause path loss to increase as the distances increase, and the receiving signal is severely distorted. Finally, in " $U$ " type measurement conditions, signal power is drastically attenuated after traveling through two walls, and the multipath signal cannot be clearly distinguished. It can be concluded that the receiver cannot receive the information effectively with an increase in the number of obstructions.

Then, we consider that the transmitter and receiver are placed in a straight tunnel that is fully occluded by the obstacles, i.e., an environment with closed glass doors is shown in Figure 3(a). The measurement setup is shown as follows. (i) The transmitter is placed at Tx1, while the receiver is placed at $\mathrm{Rx} 1$ and $\mathrm{Rx} 6$, respectively. The obtained PDPs are compared with those obtained under LOS conditions with the transmission power of $10 \mathrm{dBm}$ and distance of $20 \mathrm{~m}$, as shown in Figure 7(a). (ii) The PDPs are analyzed under different transmission powers with obstacles, while the transmitter and receiver are placed at $\mathrm{Tx} 1$ and $\mathrm{Rx} 2$, respectively, as shown in Figure 7(b). Therein, receiving SNR is $0 \mathrm{~dB}$ with the transmission power of $-20 \mathrm{dBm}$, and receiving SNR is $9 \mathrm{~dB}$ with the transmission power of $10 \mathrm{dBm}$.

Figure 7 shows that the signal is distorted as it passes through the obstacles with a low SNR. The distances of signal propagation are shorter than those in the LOS because of the presence of the obstacles. The solution is to increase the SNR to ensure efficient transmission of the signal.

Based on the above measurement results, the distinguishable multipaths in the receiver are related to SNR, Txto- $\mathrm{Rx}$ distance, and measurement scenarios. The LOS and NLOS conditions are considered when analyzing the change in distinguishable multipaths.

According to statistical results, the distinguishable multipath number can be expressed as a function within the error tolerance by using the fitting method, which is given by

$$
\mathscr{L}(d)=\left\lceil\left|\chi \exp \left(-\frac{(d-\lambda)^{2}}{\gamma^{2}}\right)-\varepsilon^{(\text {LOS/NLOS })}\right|\right\rceil,
$$

where $d>0$ in meters denotes the Tx-to- $\mathrm{Rx}$ distance, $\chi, \lambda$, and $\gamma$ denote coefficients, and $\varepsilon^{\text {(LOS/NLOS) }}$ denotes the bias.

Based on statistical measurement results, we determine the coefficients and bias as shown in Table 4. Therefore, the distinguishable multipath number can be determined using the given function.

5.3. Analysis of Multipath Power. Based on multipath results, we investigate the CDF of the multipath power in the normalized PDP, which is shown in Figure 8. The overall observation from this figure, in comparison to the NLOS condition, shows that the multipath power of the LOS condition is higher. In detail, at a CDF of $90 \%$, the multipath power is less than $-23 \mathrm{dBm}$ under the LOS condition, while that is less than $-30 \mathrm{dBm}$ under the NLOS condition. The reason is that there is no direct path, which causes that the signal attenuation is more severe under the NLOS condition.

5.4. Analysis of Multipath Arrival Delay. In channel multipath propagation, signals traveling through different paths arrive at the receiver at different times, resulting in a delay spread of the receiving signal. In general, the propagation is characterized by delay parameters including average delay spread $\bar{\tau}$ and RMS delay spread $\tau_{\text {rms }}$.

The average delay spread $\bar{\tau}$ is defined as the first order of the power delay spectrum: 


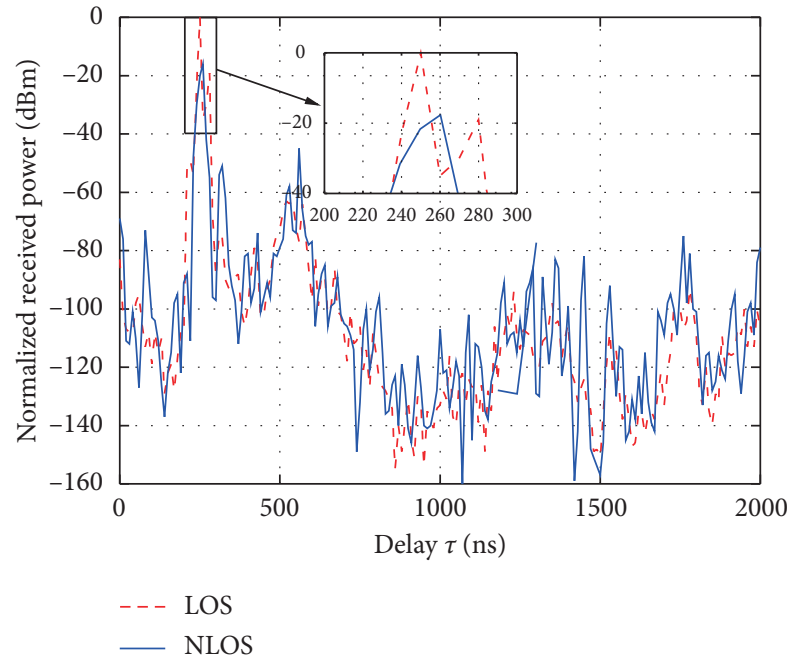

(a)

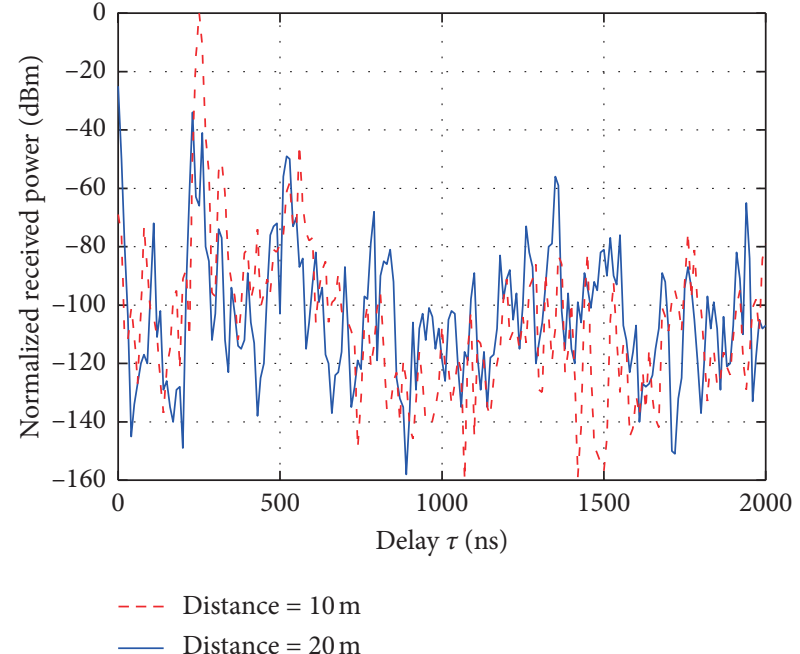

(b)

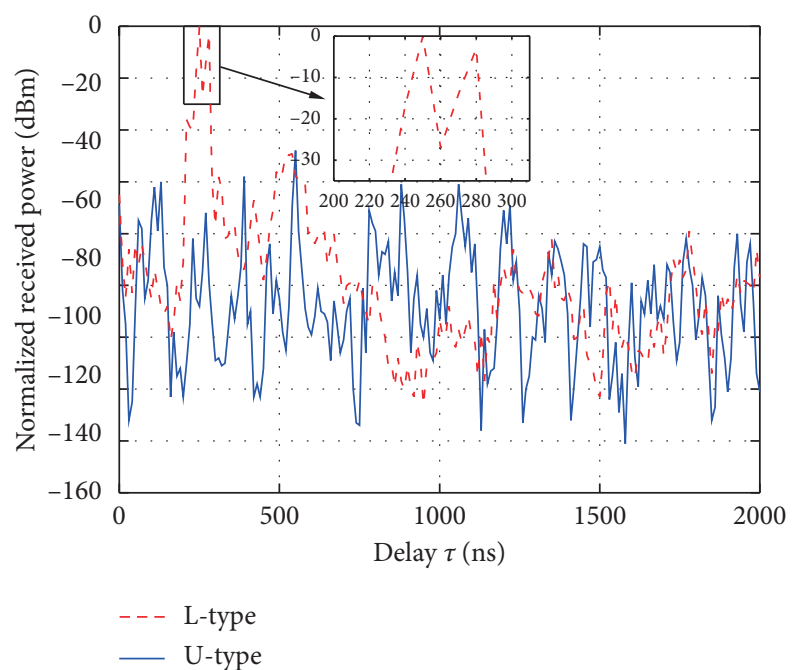

(c)

FIGURE 6: Comparison of PDPs under NLOS conditions: (a) comparison of multipath conditions under LOS and NLOS conditions with a fixed transmission power of $10 \mathrm{dBm}$ and distance of $10 \mathrm{~m}$; (b) comparison of multipath conditions with different distances; (c) comparison of multipath conditions in " $L$ " and " $U$ " type environments.

$$
\bar{\tau}=\frac{\sum_{k} p\left(\tau_{k}\right) \tau_{k}}{\sum_{k} p\left(\tau_{k}\right)}
$$

where $p\left(\tau_{k}\right)$ is the power of the $k$-th distinguishable multipath with the relative delay (the relative delay denotes the delay of the distinguishable multipath component relative to the main peak) $\tau_{k}$.
The RMS delay spread $\tau_{\text {rms }}$ is defined as the square root of the second moment of the power delay spectrum:

$$
\tau_{\mathrm{rms}}=\sqrt{\mathbb{E}\left(\tau^{2}\right)-(\bar{\tau})^{2}}
$$

where 


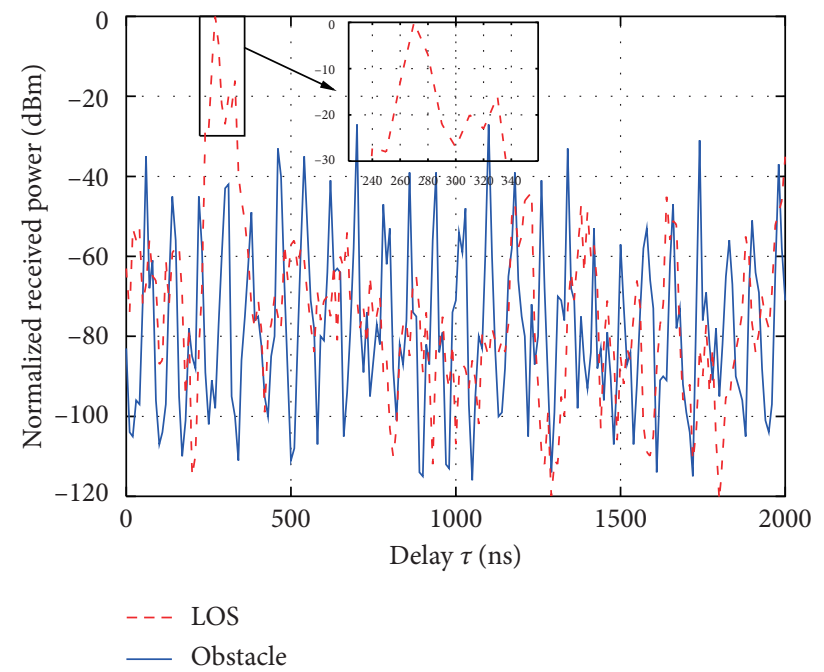

(a)

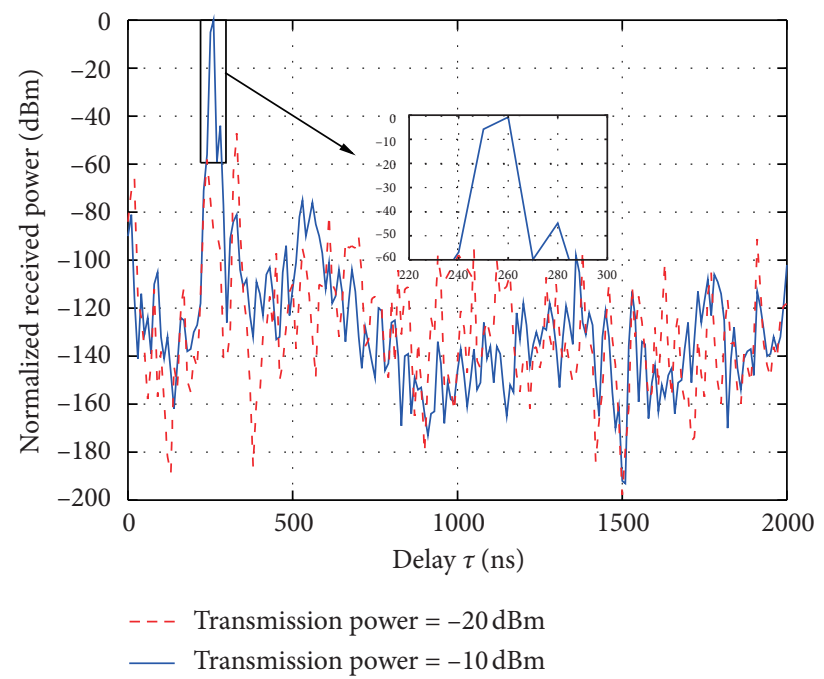

(b)

FIGURE 7: Comparison of PDPs with obstacle conditions: (a) comparison of multipath conditions under LOS and obstacle conditions with a fixed transmission power of $10 \mathrm{dBm}$ and distance of $20 \mathrm{~m}$; (b) comparison of multipaths at different transmission powers.

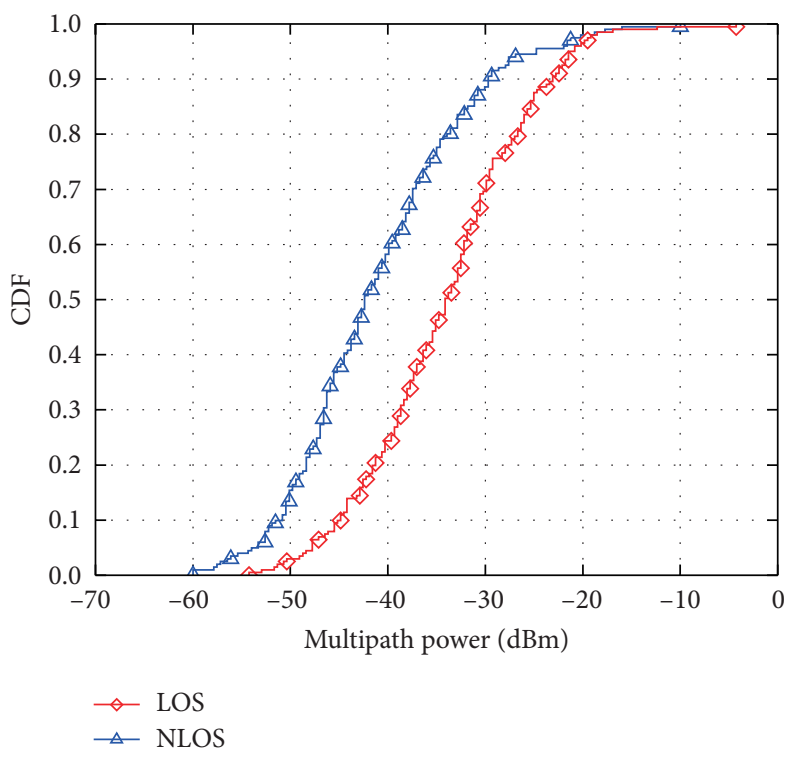

FIgURE 8: Multipath power in the normalized PDP. 


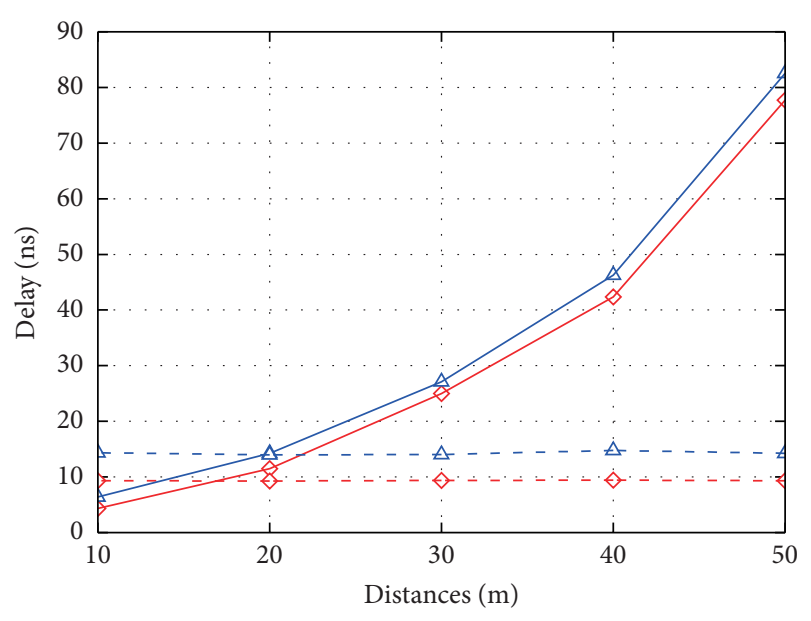

$\neg$ Average delay spread in LOS

$-\diamond-$ RMS delay spread in LOS

$\triangle \quad$ Average delay spread in NLOS

- $\Delta$ - RMS delay spread in NLOS

FIgUre 9: Delay parameters at different distances.

TABle 4: Determination of coefficients and bias.

\begin{tabular}{lccccc}
\hline Coefficients & $\chi$ & $\lambda$ & $\gamma$ & $\varepsilon^{\text {LOS }}$ & $\varepsilon^{\text {NLOS }}$ \\
\hline SNR $=10 \mathrm{~dB}$ & 3 & 30 & 10.65 & 0.06 & 1.0 \\
SNR $=20 \mathrm{~dB}$ & 4 & 50 & 10.65 & 0.06 & 1.0 \\
\hline
\end{tabular}

$$
\mathbb{E}\left(\tau^{2}\right)=\frac{\sum_{k} p\left(\tau_{k}\right) \tau_{k}^{2}}{\sum_{k} p\left(\tau_{k}\right)}
$$

Based on results of the above distinguishable multipath, channel delay parameters are analyzed at different locations under LOS and NLOS conditions, which are shown in Figure 9.

Figure 9 indicates that $\bar{\tau}$ and $\tau_{\text {rms }}$ of the NLOS are larger than those of the LOS. Moreover, the average delay spread $\bar{\tau}$ increases as the distance increases, whereas the change in the RMS delay spread $\tau_{\text {rms }}$ is not obvious. This reveals a low correlation between the RMS delay spread and the distances for both LOS and NLOS conditions [33].

\section{Conclusion}

In this paper, the SVM classifier was adopted to identify LOS and NLOS conditions. The results showed that the SVM with four conventional kernel functions can achieve a high identification accuracy. Moreover, a function of distinguishable multipath numbers was established. The coefficients of the function were determined based on statistical measurements. Furthermore, the analysis of the multipath power reveals that the multipath component signal is severely attenuated under the NLOS condition. The multipath delay parameters indicate that the average delay spread increases as the distance increases, while the RMS delay spread has a low correlation with the distance. The results of this study provided a theoretical basis for developing communication systems for tunnel environments.
For the future avenue of our works, we aim to consider more reliable and low-complexity identification methods, e.g., deep learning methods. Therefore, the multipath of the tunnel environment can be analyzed more effectively.

\section{Data Availability}

The data used to support the findings of this study can be downloaded at https://github.com/sunjc-cumt/measurementdata.

\section{Conflicts of Interest}

The authors declare that they have no conflicts of interest.

\section{Acknowledgments}

The work of Shuai Ma and Shiyin Li was supported by the National Natural Science Foundation of China (Grant nos. 61701501 and 61771474) and in part by the Shandong Provincial Major Scientific and Technological Innovation Project (MSTIP) (Grant no. 2019JZZY020505), the Open Fund of IPOC (BUPT), the Open Research Fund of National Mobile Communications Research Laboratory, Southeast University (Grant no. 2021D02), Young Talents of Xuzhou Science and Technology Plan Project (Grant no. KC19051), and the Program for "Industrial IoT and Emergency Collaboration" Innovative Research Team in CUMT (Grant no. 2020ZY002).

\section{References}

[1] K. Guan, Z. Zhong, J. I. Alonso, and C. Briso-Rodriguez, "Measurement of distributed antenna systems at $2.4 \mathrm{GHz}$ in a realistic subway tunnel environment," IEEE Transactions on Vehicular Technology, vol. 61, no. 2, pp. 834-837, 2012.

[2] S. Schwarz and M. Rupp, "Society in motion: challenges for LTE and beyond mobile communications," IEEE Communications Magazine, vol. 54, no. 5, pp. 76-83, 2016.

[3] J. Moreno, J. M. Riera, L. d. Haro, and C. Rodriguez, "A survey on future railway radio communications services: challenges and opportunities," IEEE Communications Magazine, vol. 53, no. 10, pp. 62-68, 2015.

[4] G. S. Ching, S. Nishida, A. Okuno, and Y. Kishiki, "Radio propagation simulation and measurement inside a curved and sloped subway tunnel," in Proceedings of the 2016 International Symposium on Antennas and Propagation (ISAP), pp. 210-211, IEEE, Okinawa, Japan, October 2016.

[5] A. Emslie, R. Lagace, and P. Strong, "Theory of the propagation of UHF radio waves in coal mine tunnels," IEEE Transactions on Antennas and Propagation, vol. 23, no. 2, pp. 192-205, 1975.

[6] C. Briso-Rodríguez, P. Fratilescu, and Y. Xu, "Path loss modeling for train-to-train communications in subway tunnels at 900/2400 MHz," IEEE Antennas and Wireless Propagation Letters, vol. 18, no. 6, pp. 1164-1168, 2019.

[7] J. Li and S. Wu, "Non-parametric non-line-of-sight identification and estimation for wireless location," in Proceedings of the 2012 International Conference on Computer Science and Service System, pp. 81-84, IEEE, Nanjing, China, August 2012. 
[8] D. Kim, G. Kwon, J. Pyun, and J. Kim, "NLOS identification in UWB channel for indoor positioning," in Proceedings of the 2018 15th IEEE Annual Consumer Communications Networking Conference (CCNC), IEEE, Las Vegas, NV, USA, January 2018.

[9] V. Barral, C. J. Escudero, and J. A. Garcła-Naya, "NLOS classification based on RSS and ranging statistics obtained from low-cost UWB devices," in Proceedings of the 2019 27th European Signal Processing Conference (EUSIPCO), September 2019.

[10] J. Schroeder, S. Galler, K. Kyamakya, and K. Jobmann, "NLOS detection algorithms for ultra-wideband localization," in Proceedings of the 2007 4th Workshop on Positioning, Navigation and Communication, pp. 159-166, Hannover, Germany, March 2007.

[11] C. Bonnet, H. Robert, A. M. Guyot-Hermann, and M. Thu Zin, "Improvement in performances of an apparatus testing membrane resorption in vitro, by automatic adjustment and regulation of pH," Journal de Pharmacie de Belgique, vol. 30, no. 2, 1975.

[12] G. Aceto, D. Ciuonzo, A. Montieri, and A. Pescapé, "Mobile encrypted traffic classification using deep learning: experimental evaluation, lessons learned, and challenges," IEEE Transactions on Network and Service Management, vol. 16, no. 2, pp. 445-458, 2019.

[13] G. Aceto, D. Ciuonzo, A. Montieri, and A. Pescapé, "MIMETIC: mobile encrypted traffic classification using multimodal deep learning," Computer Networks, vol. 165, 2019.

[14] S. Maran, W. M. Gifford, H. Wymeersch, and M. Z. Win, "NLOS identification and mitigation for localization based on UWB experimental data," IEEE Journal on Selected Areas in Communications, vol. 28, no. 7, pp. 1026-1035, 2010.

[15] Z. Zeng, S. Liu, and L. Wang, "UWB NLOS identification with feature combination selection based on genetic algorithm," in Proceedings of the 2019 IEEE International Conference on Consumer Electronics (ICCE), January 2019.

[16] J. B. Kristensen, M. Massanet Ginard, O. K. Jensen, and M. Shen, "Non-line-of-sight identification for UWB indoor positioning systems using support vector machines," in Proceedings of the 2019 IEEE MTT-S International Wireless Symposium (IWS), May 2019.

[17] C. L. Sang, B. Steinhagen, J. D. Homburg, M. Adams, M. Hesse, and U. Rückert, "Identification of NLOS and multipath conditions in UWB localization using machine learning methods," Applied Science, vol. 10, no. 11, 2020.

[18] C. Huang, A. F. Molisch, R. Wang, P. Tang, R. He, and Z. Zhong, "Angular information-based NLOS/LOS identification for vehicle to vehicle MIMO system," in Proceedings of the 2019 IEEE International Conference on Communications Workshops (ICC Workshops), May 2019.

[19] C. Huang, A. F. Molisch, R. He et al., "Machine learningenabled LOS/NLOS identification for MIMO systems in dynamic environments," IEEE Transactions on Wireless Communications, vol. 19, no. 6, pp. 3643-3657, 2020.

[20] R. He, Z. Zhong, B. Chen et al., "Propagation channel measurements and analysis at $2.4 \mathrm{GHz}$ in subway tunnels," IET Microwaves, Antennas \& Propagation, vol. 7, no. 11, pp. 934-941, 2013.

[21] K. Guan, B. Ai, Z. Zhong et al., "Measurements and analysis of large-scale fading characteristics in curved subway tunnels at $920 \mathrm{MHz}, 2400 \mathrm{MHz}$, and $5705 \mathrm{MHz}$," IEEE Transactions on Intelligent Transportation Systems, vol. 16, no. 5, pp. 23932405, 2015.
[22] C. Zhou, T. Plass, R. Jacksha, and J. A. Waynert, "RF propagation in mines and tunnels: extensive measurements for vertically, horizontally, and cross-polarized signals in mines and tunnels," IEEE Antennas and Propagation Magazine, vol. 57, no. 4, pp. 88-102, 2015.

[23] J. Li, Y. Zhao, J. Zhang, R. Jiang, C. Tao, and Z. Tan, "Radio channel measurements and analysis at $2.4 / 5 \mathrm{GHz}$ in subway tunnels," China Communications, vol. 12, no. 1, pp. 36-45, 2015.

[24] A. Hrovat, G. Kandus, and T. Javornik, "A survey of radio propagation modeling for tunnels," IEEE Communications Surveys \& Tutorials, vol. 16, no. 2, pp. 658-669, 2014.

[25] H. Fernández, L. Rubio, V. M. Rodrigo-Peñarrocha, and J. Reig, "Path loss characterization for vehicular communications at $700 \mathrm{MHz}$ and $5.9 \mathrm{GHz}$ under LOS and NLOS conditions," IEEE Antennas and Wireless Propagation Letters, vol. 13, pp. 931-934, 2014.

[26] M. Boutin, A. Benzakour, C. L. Despins, and S. n. Affes, "Radio wave characterization and modeling in underground mine tunnels," IEEE Transactions on Antennas and Propagation, vol. 56, no. 2, pp. 540-549, 2008.

[27] C. Cortes and V. Vapnik, "Support-vector networks," $M a$ chine Learning, vol. 20, no. 3, pp. 273-297, 1995.

[28] Z. Zeng, H. Yu, H. Xu, Y. Xie, and J. Gao, "Fast training support vector machines using parallel sequential minimal optimization," in 2008 3rd International Conference on Intelligent System and Knowledge Engineering, pp. 997-1001, Xiamen, China, November 2008.

[29] T.-W. Kuan, J.-F. Wang, J.-C. Wang, P.-C. Lin, and G.-H. Gu, "VLSI design of an SVM learning core on sequential minimal optimization algorithm," IEEE Transactions on Very Large Scale Integration (VLSI) Systems, vol. 20, no. 4, pp. 673-683, 2012.

[30] G. Wang, J. Lu, K.-S. Choi, and G. Zhang, “A transfer-based additive LS-SVM classifier for handling missing data," IEEE Transactions on Cybernetics, vol. 50, no. 2, pp. 739-752, Feb. 2020.

[31] C. J. C. Burges, "A tutorial on support vector machines for pattern recognition," Data Mining and Knowledge Discovery, vol. 2, no. 2, pp. 121-167, 1998.

[32] Recommendation ITU R P.1407 Multipath Propagation and Parameterization of is Characteristics, http://www.itu.int.

[33] S. Li, Y. Liu, L. Lin, Z. Chen, X. Zhang, and X. Zhu, "Measurement and simulation of wideband channel characterization in the underground tunnel environment," in Proceedings of the 2017 Sixth Asia-Pacific Conference on Antennas and Propagation (APCAP), Xi'an, China, October 2017. 